\title{
Synthetic Elaboration of Native DNA by RASS (SENDR)
}

\author{
Dillon T. Flood, ${ }^{1 \perp}$ Kyle W. Knouse, ${ }^{\perp \perp}$ Julien C. Vantourout, ${ }^{1}$ Brittany B. Sanchez, ${ }^{\S}$ Emily J. \\ Sturgell, ${ }^{\S}$ Jason S. Chen, ${ }^{\S}$ Phil S. Baran,$^{1^{*}}$ Philip E. Dawson. ${ }^{*}$
}

${ }^{1}$ Department of Chemistry, Scripps Research, 10550 North Torrey Pines Road, La Jolla, California 92037, United States $\S$ Automated Synthesis Facility, Scripps Research, 10550 North Torrey Pines Road, La Jolla, CA 92037, United States

$\perp$ These authors contributed equally to this work.

\begin{abstract}
The controlled, site-specific ligation of molecules to native DNA remains an unanswered challenge. Herein, we report a simple solution to achieve this ligation through the tactical combination of two recently developed technologies: One for the manipulation of DNA in organic media, and another for the chemoselective labeling of alcohols. Reversible Adsorption of Solid Support (RASS) is employed to immobilize DNA and facilitate its transfer into dry acetonitrile. Subsequent ligation with $\mathrm{P}(\mathrm{V})$-based $\Psi$ reagents takes place in high yield with exquisite selectivity for the exposed 3' or 5' alcohols on DNA. This two-stage process, dubbed SENDR for Synthetic Elaboration of Native DNA by RASS, can be applied to a multitude of DNA conformations and sequences with a variety of functionalized $\Psi$ reagents to generate useful constructs. Such entities can address numerous longstanding challenges, including the selective single coupling of DNA to proteins, ASOs, and functional small molecules, and also can allow the synthesis of doubly-labeled congeners for novel probe constructs including ones of potential interest to COVID-19 research. Finally, a prototype for the industrialization of SENDR in a kit format is presented.
\end{abstract}

\section{Introduction}

DNA conjugates are ubiquitous in the fields of chemical biology, biophysics, and diagnostics (Figure 1A). ${ }^{1}$ Indeed, DNA conjugate-based technology has provided the basis for many modern technological advances. ${ }^{1}$ For example, DNA-PAINT conjugates enable transforming super resolution microscopy, ${ }^{2-5}$ and TaqMan PCR probes have revolutionized precision diagnostics. $^{6-11}$ These hybridization probes all require custom DNA oligomer conjugates that are precisely functionalized and homogeneous. ${ }^{1}$ Clearly, such homogeneous functionalization is a challenge of the highest magnitude for chemoselective chemical ligation.

Hybridization probes operate through the exquisite molecular recognition ability that a single strand of DNA displays towards its complimentary sequence. ${ }^{1,12,13}$ The ability for DNA to take on well-defined conformations, set by inter- and intramolecular interactions, allows for another dimension of selectivity. ${ }^{1,14,15}$ Detecting these molecular interactions usually requires the incorporation of a fluorophore or radioactive moiety. ${ }^{1}$ Thus, DNA-probe conjugates form the foundation that allows for ubiquitous biochemical and diagnostic techniques such as

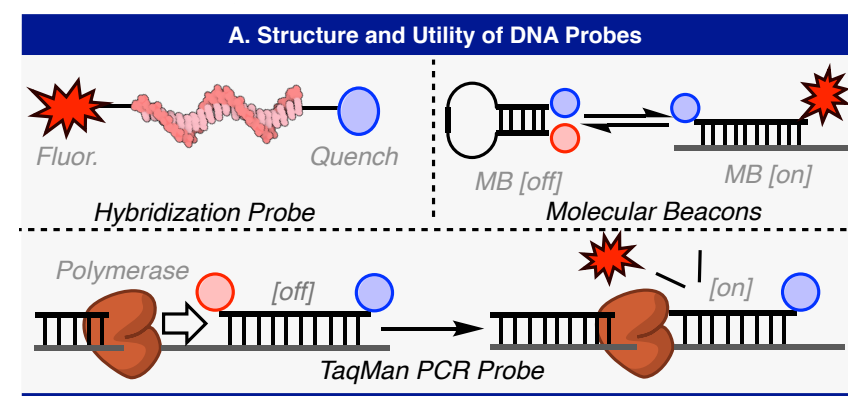

B. Random Labelling of DNA: Enzymatic and Chemical Methods

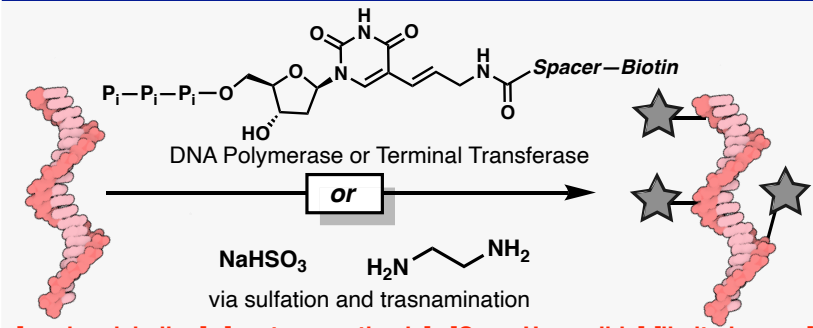

[random labeling] [custom synthesis] [C $\rightarrow$ U possible] [limited scope]

C. Site Specific DNA Modification: Phosphoramidate Formation

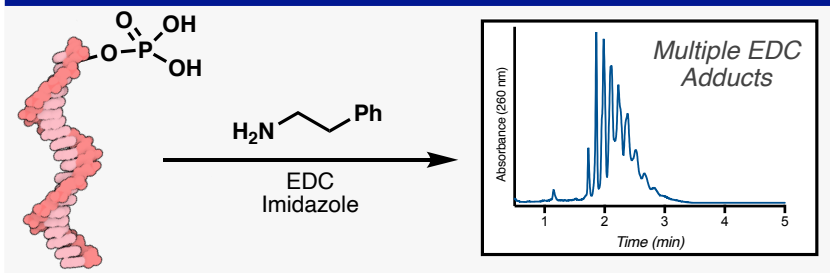

[non-homogeneous activation] [many by-products] [limited scope]

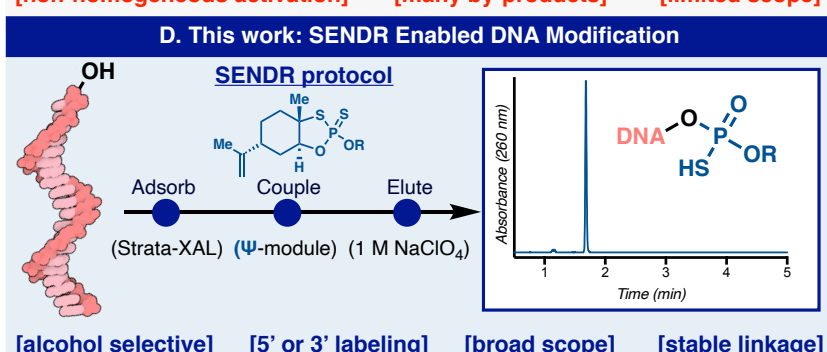

Figure 1. Modification of native DNA. (A) Structure and utility of some DNA hybridization probes (B) Random labeling of DNA through enzymatic incorporation of modified bases and chemical methods. (C) Chemical modification of DNA: Phosphoramidate formation. (D) This work: SENDR. 
Southern ${ }^{16}$ and Northern blotting, ${ }^{17}$ molecular beacons, ${ }^{1,15}$ and TaqMan qPCR. ${ }^{6-11}$

Although these techniques are universal, chemical synthesis of the requisite DNA conjugates can be cost-prohibitive and laborintensive. ${ }^{1}$ As a result, less sensitive techniques not requiring precision-labelled DNA are often utilized (Figure 1B). ${ }^{1}$ As DNA tagging becomes increasingly popular, massively multiplexed experiments would require thousands of chemically synthesized and modified oligonucleotides. Thus, novel ways to cheaply, precisely, and simply build DNA conjugates, ideally from ubiquitous substrate building blocks, would be of great interest to researchers across many fields.

\section{Chemical Approach Site-Specific DNA Modification}

Although many chemical and biochemical methods exist for the random labeling of native DNA, all methods produce nonhomogenous products that contain modified bases determined randomly or statistically (Figure $1 \mathrm{~B}$ and C). ${ }^{18-21}$ In some cases, DNA with randomly-incorporated labels can be useful, but unlocking the total power of DNA as a molecular recognition probe requires site-selective incorporation of the desired tag.

One classic method for selective modification of native DNA exists, although it presents with several limitations. The method first reported in 1983 by Orgel relies on a water-soluble carbodiimide (EDC), imidazole, and amines, which when combined furnish a phosphoramidate linkage between native DNA bearing a 5' phosphate group and an amine of interest. ${ }^{22-26}$ Although this method is widely cited, its scope is limited to simple amines, and the reaction and workup must be conducted rapidly to avoid premature hydrolysis of the carbodiimide. In our hands, this method proved unreliable, providing a product that was contaminated with many EDC adducts, even after multiple attempts varying concentration and stoichiometry (Figure 1C, and also see SI).

In an attempt to remedy this, we set out to combine two technologies previously disclosed from our respective (P.S.B and P.E.D.) laboratories. Specifically, P(V)-based $\Psi$ reagents have been established to construct stereochemically-pure phosphorothioate linkages between hydroxyl nucleophiles in a chemoselective fashion (Figure 1D). ${ }^{27}, 28$ While upon first glance, it seemed simple to employ this reagent system for the labeling of DNA 5' or 3' hydroxyl groups, $\Psi$-loaded reagents are typically employed in dry acetonitrile, as they are readily hydrolyzed in water. $^{27,} 28$ In contrast, due to the highly charged nature of the native DNA phosphate backbone, these polymeric substrates are insoluble in most organic solvents and usually require significant water content to solubilize it in a mixed aqueous/organic system. ${ }^{29,} 30$ These mutually-exclusive properties precluded the simple adaptation of $\Psi$ for the purposes of site-specific DNA labeling. Reversible Adsorption of Solid Support (RASS), an alternate paradigm for performing chemistry on-DNA, thus seemed like an ideal merger with $\Psi$-based chemistry. RASS is a process which allows for the adsorption of biomacromolecules onto a solid support to facilitate their transfer into solvents or reaction paradigms that would previously be considered incompatible. ${ }^{31-34}$ In this manifestation, DNA is adsorbed to a polystyrene-based cationic support, through a simple mixing procedure, and the solvent exchanged (by simple washing and drying) into near-anhydrous conditions. In turn, waterincompatible reactions are enabled. Herein, we describe the union of $\Psi$ and RASS for the site specific labeling of oligonucleotides, Synthetic Elaboration of Native DNA by RASS: SENDR.

With this design in mind, a variety of $\Psi$-loaded reagents ( $\Psi$ modules, Figure 2) were prepared to explore site-specific labeling of native DNA. Model DNA (1) and (2) each contain single modifiable terminal hydroxyl groups at the 5' and 3' positions, respectively, with the other terminal hydroxyl group capped with a phosphate group (Figure 3A). Model DNA could readily undergo RASS (Strata AXL resin, Phenomenex), but initial attempts to apply $\Psi$-conjugation proved difficult, furnishing only low yields of the modified product. Based on significant amounts of hydrolyzed $\Psi$-derivatives in the crude product mixture, we postulated that this protocol ( $3 \mathrm{X}$ washes with dry acetonitrile) was not sufficient to fully dry the DNA-bound resin. Residual water would subsequently quench the $\Psi$-modules upon addition of DBU. ${ }^{28}$ Optimization of the washing protocol to use reagent grade DMA, then THF, and finally drying under vacuum rectified this issue. With this revised protocol in hand, promising initial reactivity was observed (Figure $3 \mathrm{~B}$, entry 1 ). By modulating DBU stoichiometry, concentration, and reaction time, general conditions were identified (Figure $3 \mathrm{~B}$, entry 3 ) to produce singlylabeled products $\mathbf{3}$ and $\mathbf{4}$ with good conversion (determined by total UV quantification at $260 \mathrm{~nm}$ ) at both the 5' and 3' position, with no observable by-products. The labeling position was confirmed by MS fragmentation (See SI). The conditions also proved to be sequence independent (Figure 3C). SENDR provided efficient ligations to oligonucleotides regardless of the identity of the terminal nucleoside. Also, both "sticky ends" (i.e., overhanging oligonucleotides) and "blunt ends" (i.e., nonoverhanging oligonucleotides) could be modified efficiently. With all of these elements combined, the development of SENDR was complete and our attention turned to its application. Based on previous studies, it was envisioned that a wide array of $\Psi$ modules derived from alcohol nucleophiles could be prepared as stable (and often crystalline) reagents. ${ }^{27,28}$ Combined with the present findings, subsequent coupling to DNA sequences would allow for a vast scope. Indeed, a large number of $\Psi$-modules (Figure 2, $\Psi-1-\Psi-13)$, including multiple click chemistry handles (19-24, 36-37), protected amines (17-22, 27-28), an activated disulfide (29-30), an MRI probe (25-26), a fluorescent quencher (33-34), a ligand for radiomedicine (35), photoaffinity tag (2122), and nucleosides (31-32, 38-39), were prepared (Figure 3D). All $\Psi$-modules produced singly labeled products upon ligation to DNA in good to excellent conversion at both the 3' and 5' hydroxyl groups. DNA recovery was also good (50-80\%), given that the upper yield limit after ethanol precipitation is known to be

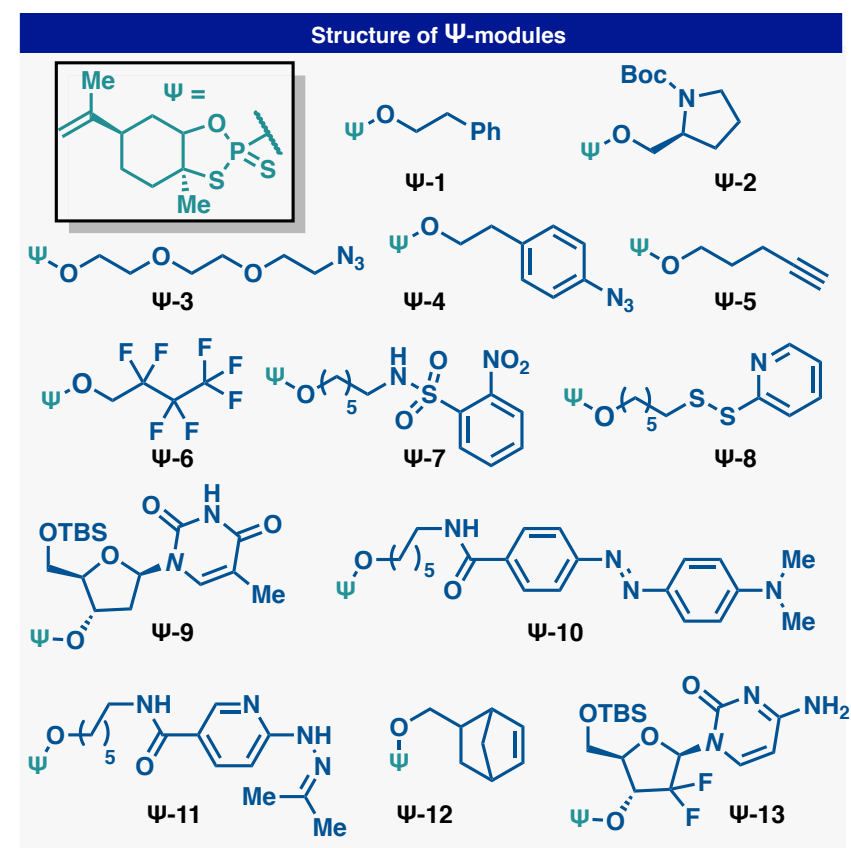

Figure 2. $\Psi$-modules synthesized for this study. 


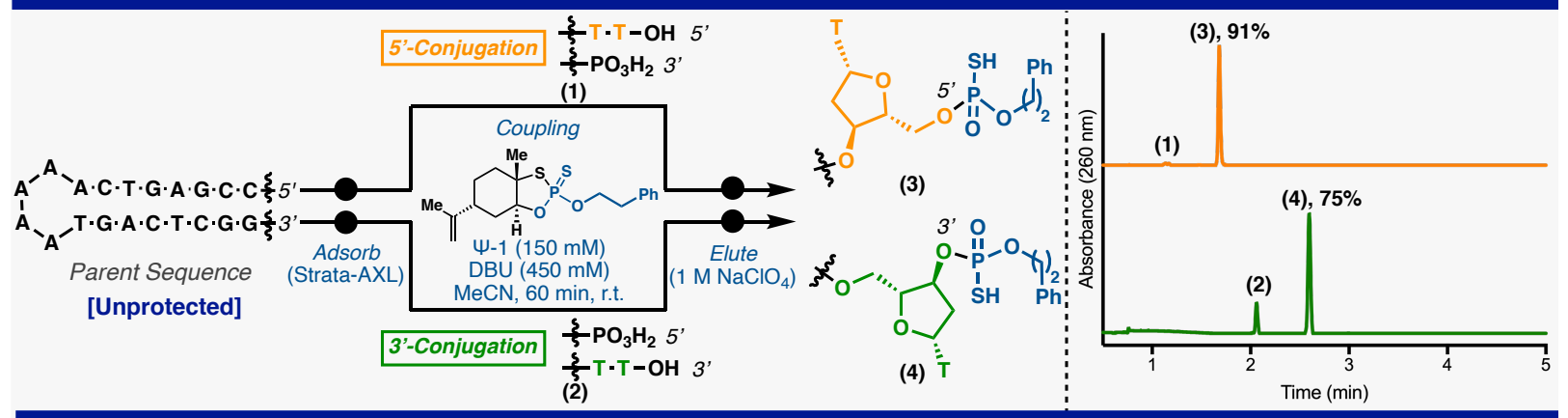

\begin{tabular}{|c|c|c|c|}
\hline Entry & Conditions & (3) Conversion (\%) & (4) Conversion (\%) \\
\hline 1 & $50 \mathrm{mM} \Psi-1,150 \mathrm{mM}$ DBU, $60 \mathrm{~min}$ & 56 & 17 \\
\hline 2 & $100 \mathrm{mM} \Psi-1,300 \mathrm{mM}$ DBU, $60 \mathrm{~min}$ & 72 & 51 \\
\hline 3 & $150 \mathrm{mM} \Psi-1,450 \mathrm{mM} \mathrm{DBU}, 60 \mathrm{~min}$ & 91 & 72 \\
\hline 4 & $150 \mathrm{mM} \Psi-1,450 \mathrm{mM} \mathrm{DBU}, 60 \mathrm{~min} \times 2$ & 84 & 62 \\
\hline 5 & $150 \mathrm{mM} \Psi-1,450 \mathrm{mM}$ DIPEA, $60 \mathrm{~min}$ & 0 & 0 \\
\hline 6 & $150 \mathrm{mM} \Psi-1,450 \mathrm{~m}$ NMe-Morpholine, $60 \mathrm{~min}$ & 0 & 0 \\
\hline 7 & $150 \mathrm{mM} \Psi-1,450 \mathrm{mM}$ 2,6-Lutidine, $60 \mathrm{~min}$ & 0 & 0 \\
\hline 8 & $150 \mathrm{mM} \Psi-1,450 \mathrm{mM}$ DBU, $60 \mathrm{~min}$, Without Stringent Drying & $<10 \%$ & $<10 \%$ \\
\hline 9 & $150 \mathrm{mM} \Psi-1,450 \mathrm{mM} \mathrm{DBU}, 60 \mathrm{~min}, 1 \%$ Water & $<10 \%$ & $<10 \%$ \\
\hline 10 & $150 \mathrm{mM} \Psi-1,450 \mathrm{mM}$ DBU, $60 \mathrm{~min}$, Without Resin & 0 & 0 \\
\hline
\end{tabular}

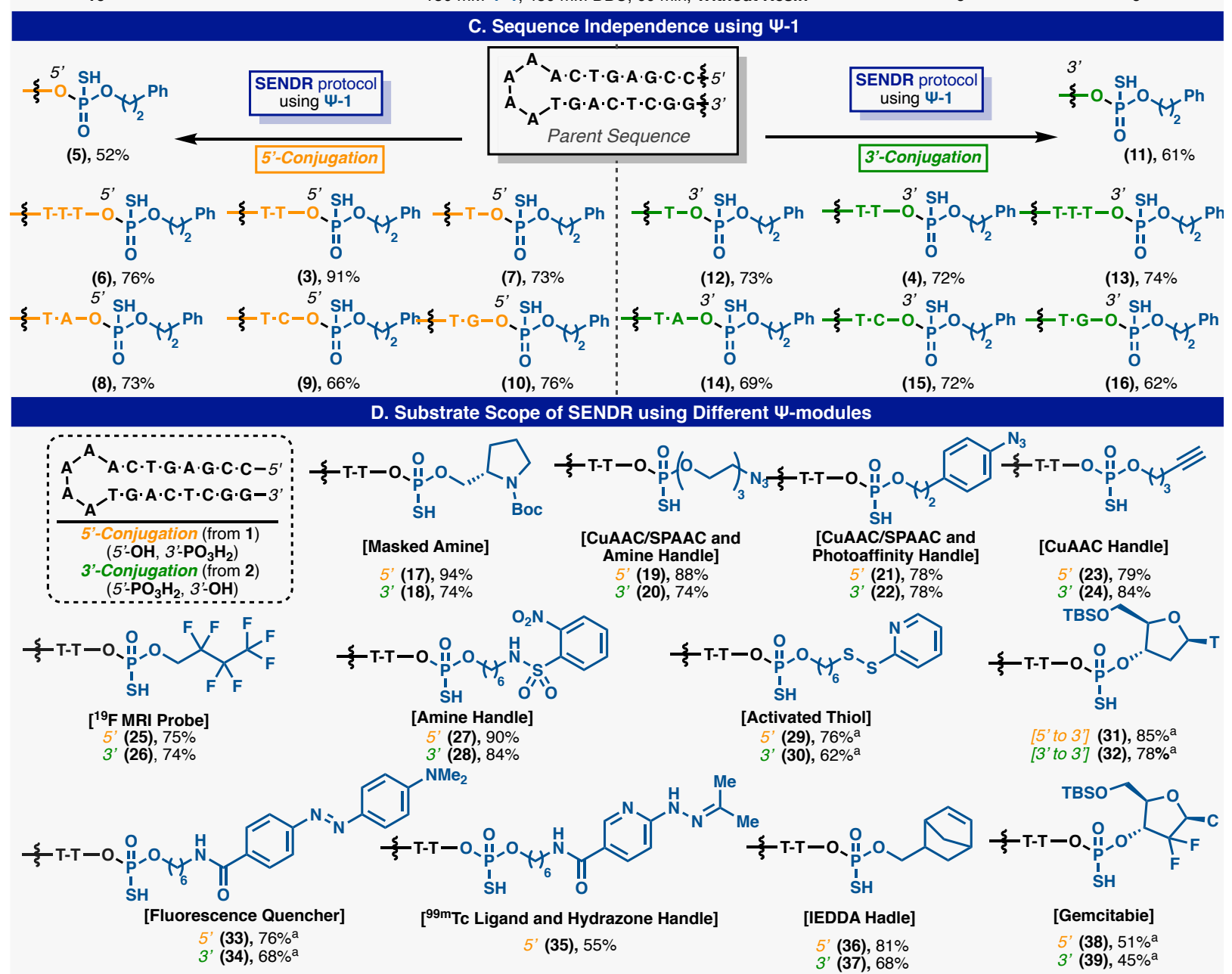

Figure 3: $\mathrm{P}(\mathrm{V})$ based DNA modification. (A) SENDR enabled DNA modification. (B) Optimization of the coupling step. (C) Sequence independence. (D) Substrate scope. Conversions based on HPLC integration of total absorbance signal at $260 \mathrm{~nm}$. Unless otherwise noted standard reaction conditions were applied; $\Psi$-module $(150 \mathrm{mM}), \mathrm{DBU}(450 \mathrm{mM})$, in dry MeCN $(250 \mu \mathrm{L}), 60 \mathrm{~min}$, r.t. while adsorbed to Strata XAL. areaction performed at $37^{\circ} \mathrm{C}$. DNA loading (adsorption step) preformed in PBS. Resin washed with DMA (x2) and THF (x3). Resin dried under vacuum $2 \mathrm{hr}$. Elution was performed using elution buffer 1 M NaClO4, 40 mM $\quad$ Tris $\quad \mathrm{pH} \quad 8.5, \quad 20 \% \quad \mathrm{MeOH}$. 
$80-85 \% .^{35}$ Throughout these applications, the general conditions were not modified, with the exception of cases where solubilization of the reagent required slightly elevated temperature $\left(37^{\circ} \mathrm{C}\right)$. Unfortunately, reagents containing extremely lipophilic substituents, such as cholesterol and oleyl alcohol, provided lower yields $(<50 \%)$ under these conditions (See SI).

The SENDER-modified oligonucleotides could be further processed via additional ligation and click manipulations (Figure 4). Thus, DNA-linked azides and alkynes were competent in $\mathrm{SPAAC}^{36-38}$ and $\mathrm{CuAAC}^{36,} 37,39$ respectively, and directly provided constructs that were useful without further purification. In addition, DNA-linked azides could be easily transformed into the corresponding amines through the addition of a water-soluble phosphine (TCEP). ${ }^{40}$ This manipulation could be performed after SENDR as a one-pot procedure in the elution buffer, providing an

\section{SENDR: Secondary Manipulations}

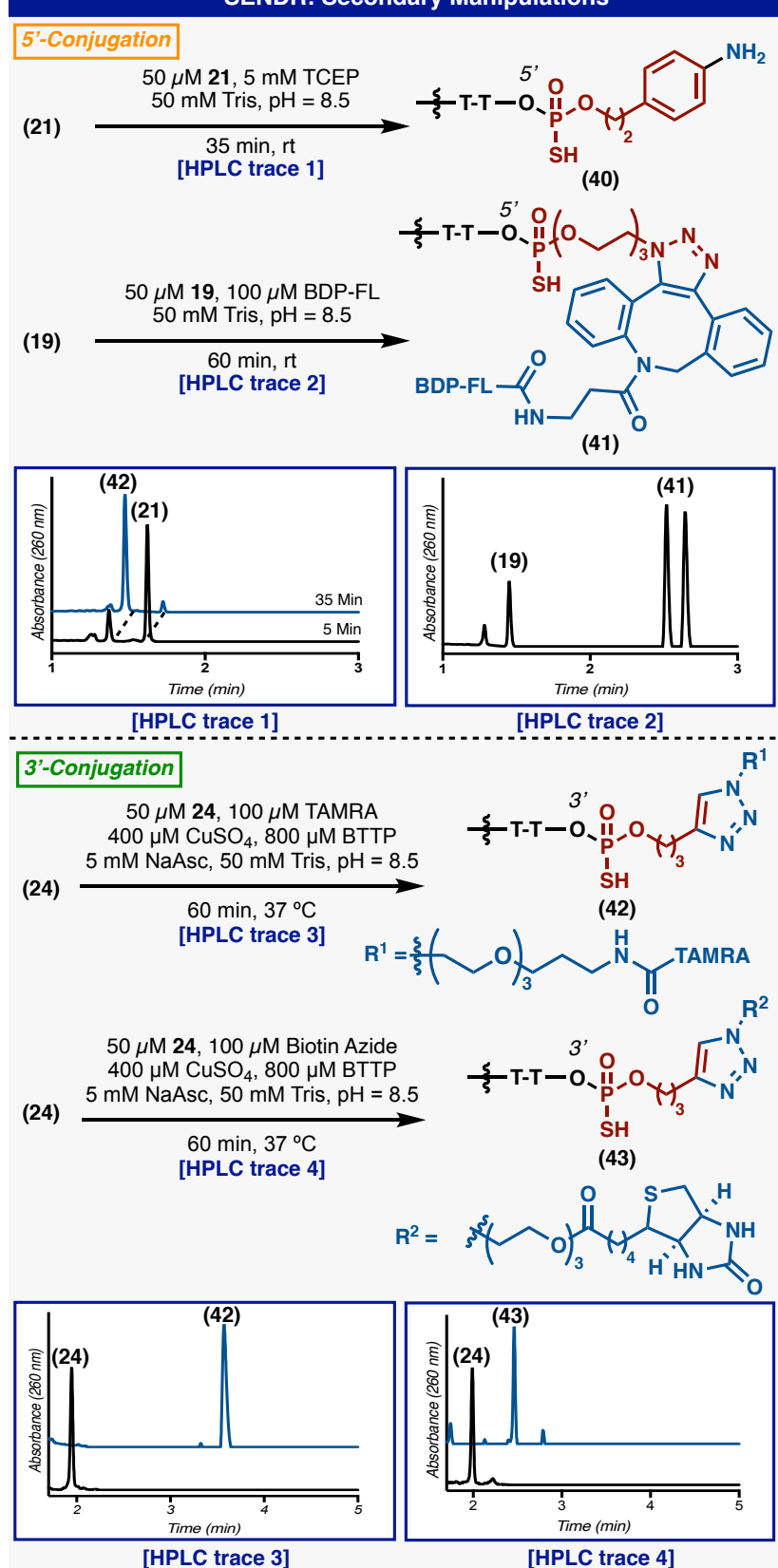

Figure 4: Downstream synthetic manipulations of SENDR-derived DNA-small molecule hybrids (ligated at 3' or 5'). exceedingly simple route to amine-modified DNA. Similarly, the construction of high-value DNA labeled with 3' TAMRA or biotin could be accomplished through bioorthogonal CuAAC chemistry. The 3' SENDR-modified DNA could be quantitatively transformed with multiple complex azides to furnish DNA conjugates that are sufficiently homogenous $(>80 \%)$ for most biochemical experiments without additional purification. Potential adverse effects on $\mathrm{Cu}(\mathrm{I})$ chemistry that could arise, due to coordination to the phosphorothioate moiety, ${ }^{41}$ were not observed and $\mathrm{CuAAC}$ could be readily employed, allowing for, in principle, near-infinite diversification.

It is worth noting that SENDR not only facilitates access to useful DNA assemblies but also considerably reduces the cost of their fabrication with minimal labor. To put these cost savings in perspective, it would cost the practitioner more than \$600 (2020 catalog pricing) to use a similar construct to 43 , whereas SENDR would deliver it for less than \$30. A similar trend is observed with compound 42, with a $\$ 730$ difference in price between the commercially-available probe and the analogous one prepared using SENDR ( $\$ 800$ vs. \$30). These significant savings combined with SENDR's ease of use and generality could facilitate ultrahigh throughput experiments by democratizing access to a broad array of DNA-linked constructs.

\section{SENDR: Complex settings}

SENDR could also be adapted for the efficient modification of both phosphorothioate antisense oligonucleotides (ASOs) and large structured aptamers (Figure 5). The 3' phosphorylated version of Vitravene, an FDA-approved ASO for the treatment of cytomegalovirus retinitis $(\mathrm{CMV})^{42,}{ }^{43}$ was efficiently ligated with a number of $\Psi$-modules with no change to the general protocol.

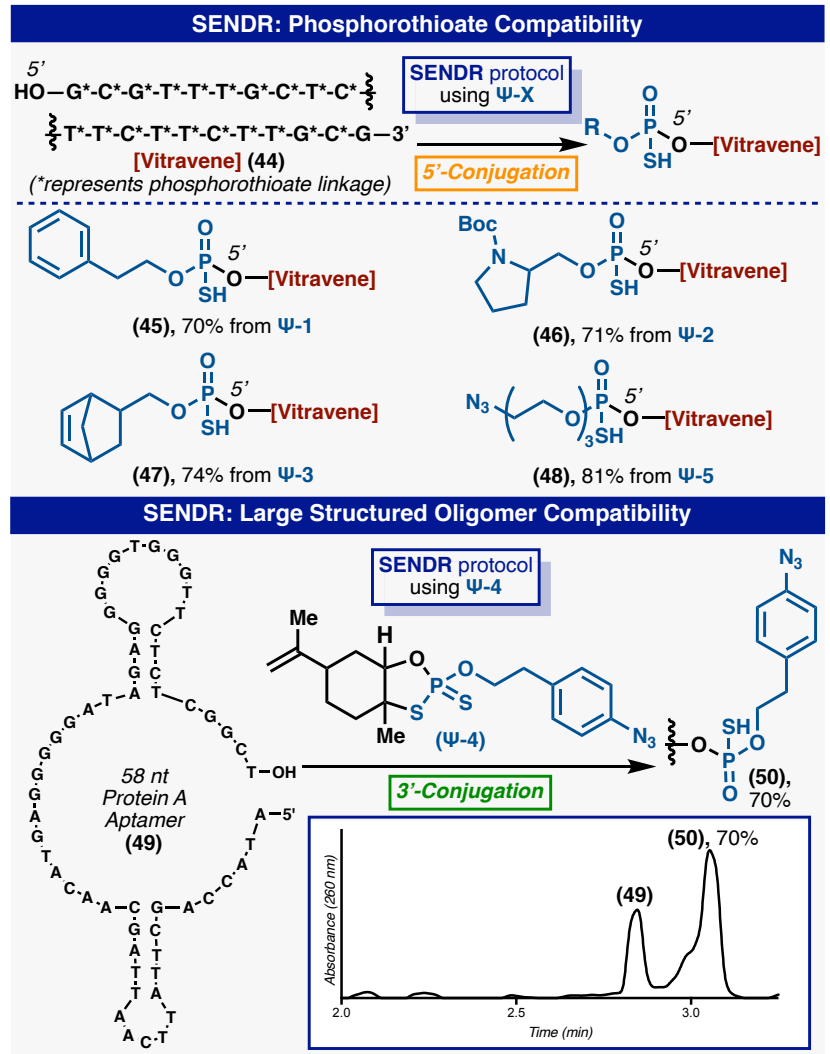

Figure 5: (A) SENDR compatibility with PS DNA (B) SENDR compatibility with larger structured oligomers. Standard reaction conditions were applied; $\Psi$-module $(150 \mathrm{mM})$, DBU $(450 \mathrm{mM})$, in dry $\mathrm{MeCN}(250 \mu \mathrm{L}), 60 \mathrm{~min}$, r.t. while adsorbed to Strata XAL. 
This result exemplifies the opportunity for the late-stage modification of ASO pools with target-engaging small molecules or peptides. Previously, such handles would have required a de novo chemical synthesis for each new compound. Also, a large (58 nt) "protein A" aptamer ${ }^{44}$ which exhibits significant secondary structure could also be modified at the 3' hydroxyl in $70 \%$ conversion. This example further demonstrates the sequence and structure independence of the SENDR platform. It could prove useful in the modification of entire SELEX ${ }^{45,46}$ pools with libraries of reactive warheads or target engaging moieties for the facile creation of DNA-small molecule chimeric inhibitors.

\section{SENDR: DNA-protein conjugates}

Another utility of SENDR was also demonstrated in the formation of DNA-protein conjugates, which are becoming increasingly valuable in the production of long-acting and/or targeted oligonucleotide drugs. ${ }^{47-50}$ An oligonucleotide was ligated by SENDR (using $\Psi$-module 6 ) with an activated disulfide group resulting in $\mathbf{5 2}$ (Figure 6A). This construct could be used directly, without purification in a disulfide forming reaction with bovine serum albumin (BSA). The reaction cleanly furnished the DNA-BSA conjugate (53) and ESI-TOF analysis of the crude reaction mixture indicated that no unmodified BSA remained in solution. ${ }^{51,52}$ Conjugation to serum albumin is a valuable half-life increasing strategy that is commonly employed in the context of readily-cleared peptide and small protein drugs, and could in principle be applied to next-generation ASOs. ${ }^{53-57}$

SENDR was also used to create a DNA construct that could be used in site-specific antibody conjugations (Figure 6B). The complementary cDNA sequence of FDA-approved ASO Tegsedi ${ }^{58-60}$ (cTegsedi) was modified with an alkyne handle. In turn, this alkyne was ligated to a reactive beta-lactam containing moiety via $\mathrm{CuAAC}$. The beta lactam containing oligonucleotide (55) was competent in the site-specific labeling of an engineered lysine on the heavy chain of a dual variable domain (DVD) IgG that has been pioneered for use in antibody drug conjugates
(ADCs) by the Barbas and Rader labs. ${ }^{61-64}$ This system was derived from the anti-hapten $\mathrm{mAb} \mathrm{h} 38 \mathrm{C} 2$ and is especially reactive toward beta lactam haptens. The reactive lysine residue also catalyzes a retro aldol reaction with methodol, which results in increased fluorescence of the aldehyde product. ${ }^{61-64}$ The modified site on the DVD was confirmed to be the catalytic lysine via methodol florescence assay - after ligation, signal from the florescent aldehyde was not detected (Figure 6B). ${ }^{61-64}$ These DVDs have shown promise as flexible platforms for the production of antibody drug conjugates, as they can be produced by typical recombinant methods, and drug molecules can be added at a known stoichiometry, to a known position, through a stable amide linkage. ${ }^{61-64}$ Labeling the DVD with cTegsedi created, in effect, an ASO delivery system that could protect, target and deliver Tegsedi to the cell of interest. This process may be useful in the creation of many antibody-ASO conjugates that could provide targeted ASO therapies. The above two examples enabled by SENDR are striking due to the ease and efficiency with which these complex conjugates could be prepared, along with the near-infinite flexibility in design.

\section{SENDR: Dual Labeled Probes}

Although many DNA based technologies only require a single probe, the true power of hybridization probes is realized in dual labeled form. ${ }^{1}$ The canonical dual labeled DNA probe has a fluorophore label on one terminus and a fluorescence quencher at the other (Figure 7A). ${ }^{1}$ Fluorescence of the probe is quenched when the two components are in close proximity. Molecular beacons (MBs) ${ }^{15}$ for example, form a stem loop system which brings the termini labels into close proximity when the target is not present (Figure 1A). Upon target engagement, the MB adopts an extended conformation, which moves the two labels out of FRET range and results in a fluorescence signal. ${ }^{15}$ Another ubiquitous example of dual labeled probes is the TaqMan qPCR probe. ${ }^{6-11}$ These probes are also typically constructed with a fluorophore and a quencher at the termini. ${ }^{6-11}$ These probes

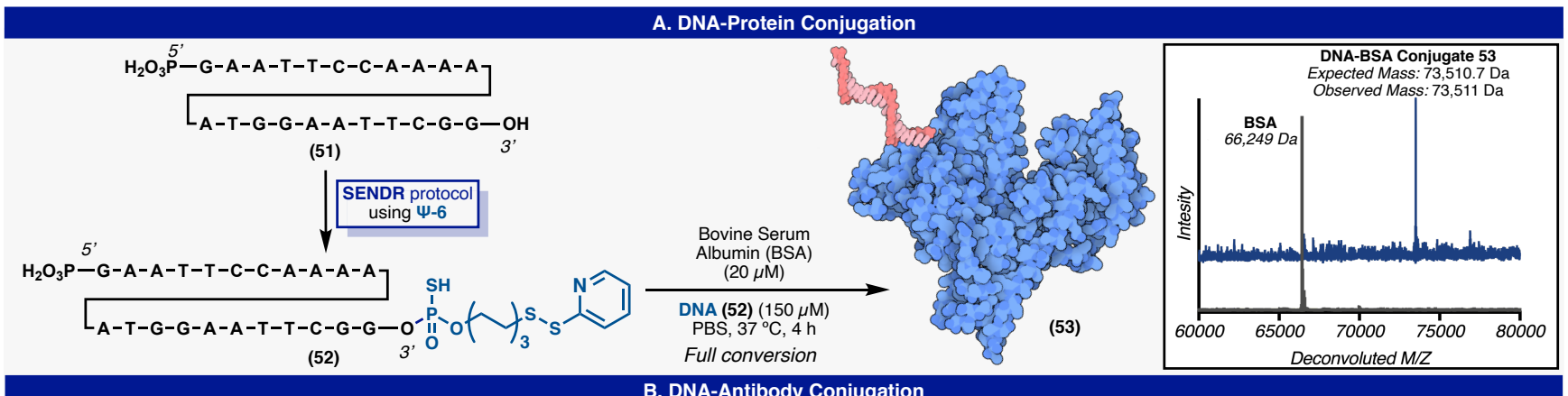

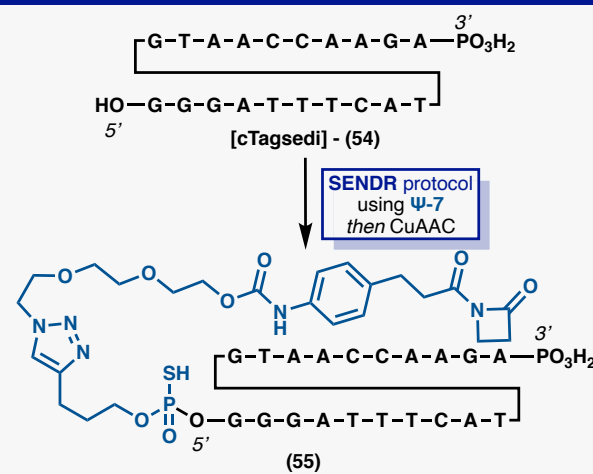

(55)

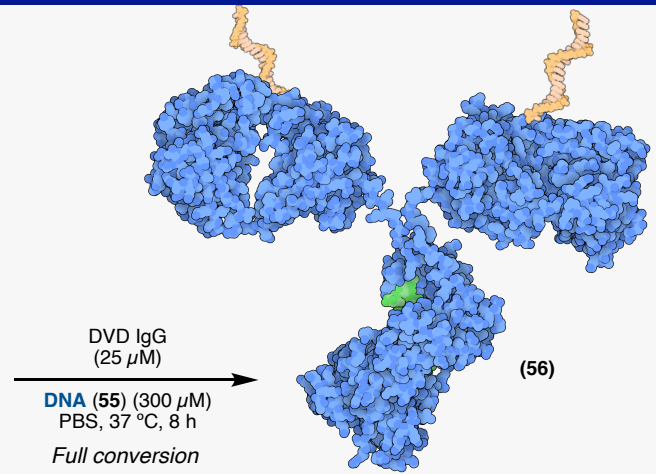

Full conversion

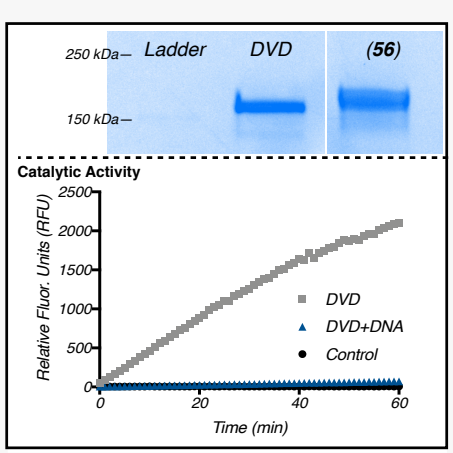

Figure 6: SENDR enabled DNA-protein conjugation. (A) DNA-BSA conjugation (ESI-ToF mass spectra of starting material and product). (B) cTegsedi-DVD conjugation (SDS Page Gel and catalytic methodol fluorescence assay including control experiments). 
hybridize to a diagnostic sequence of interest and upon PCR elongation by Taq polymerase, the probe is cleaved (by the intrinsic exonuclease activity of Taq) and increased fluorescence is read out (Figure 1A). ${ }^{6-11}$ Although TaqMan PCR is widely considered to be the state-of-the-art in real-time PCR methods, practitioners are reliant on vendors for custom synthesis of probes with proprietary linking technologies. This synthesis must be done for each individual target and can prove to be prohibitively expensive. Indeed, the less sensitive method of SYBR Green based qPCR, which relies on increased fluorescence of an intercalating dye during polymerization, is gaining in popularity because of the immense cost of buying custom TaqMan PCR probes for every experiment.

SENDR, when used in concert with ubiquitous biochemical techniques, presents a unique opportunity for a biochemical researcher to produce dual labeled probes for their own custom applications (Figure 7A). Synthesis of these probes proceeds through a three-stage process. In the event, a typical synthetic oligonucleotide containing a 5 ' phosphate (the modification that would result from biochemical production) and a 3' hydroxyl group is ligated with an azide group by SENDR (Figure 6B). Next, this modified oligomer is quantitatively dephosphorylated with recombinant Shrimp Alkaline Phosphatase (rSAP) ${ }^{65}$ to unmask the 5' hydroxyl group. Finally, this oligomer is subjected to a second SENDR modification at the 5' terminus, providing the dual probe in $65 \%$ crude conversion (Figure $7 \mathrm{~B}$ ). The position of each label on the dual labeled probe was confirmed by MS fragmentation (See SI). This dual-labeled parent probe $\mathbf{6 0}$ is now primed for subsequent SPAAC/CuAAC reactions with any fluorophore/quencher pair desired to furnish the qPCR-competent probe. Aside from the obvious advantages of simple in-house production of a user-selected array of constructs, massive cost and time savings can be realized by the typical researcher. A typical probe costs upwards of $\$ 2000$ dollars at time of this writing, and it can take over a week to synthesize. In contrast, the SENDRderived probe would cost around $\$ 35$ to produce and can be generated within 2 days. The utility of in-house probe production was demonstrated by the facile and expedient production of dual labeled probes for COVID-19 diagnostics (Figure 7C). The native sequences for the panel of RT-PCR probes for COVID-19 diagnostics (as defined by HHS 24 Jan 2020) ) $^{66,67}$ were transformed in parallel ( $\sim 48 \mathrm{hrs}$ ) into dual labeled probes through the above sequences in good overall conversions. In times of pandemic, such as COVID-19, when the commercial capacity for diagnostic probe creation is being shuttled towards the clinic, SENDR could provide researchers with a valuable alterative probe source allowing continued therapeutic and vaccine research.

\section{A. Dual Labelled Probe: An expensive tool from a cheap DNA precursor}

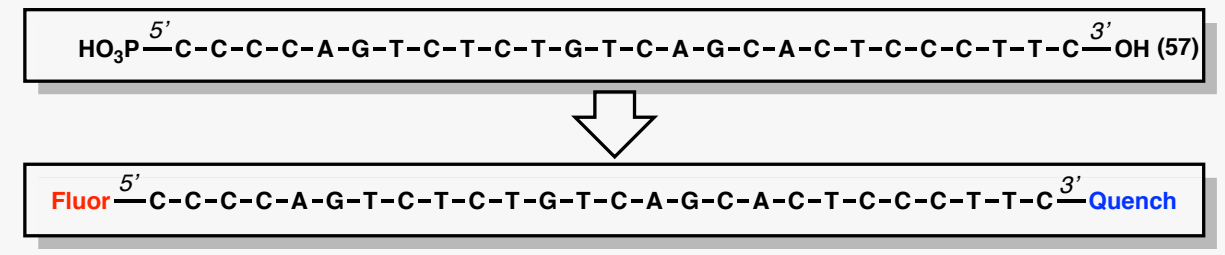

B. SENDR enabled the synthesis of a dual labeled probe
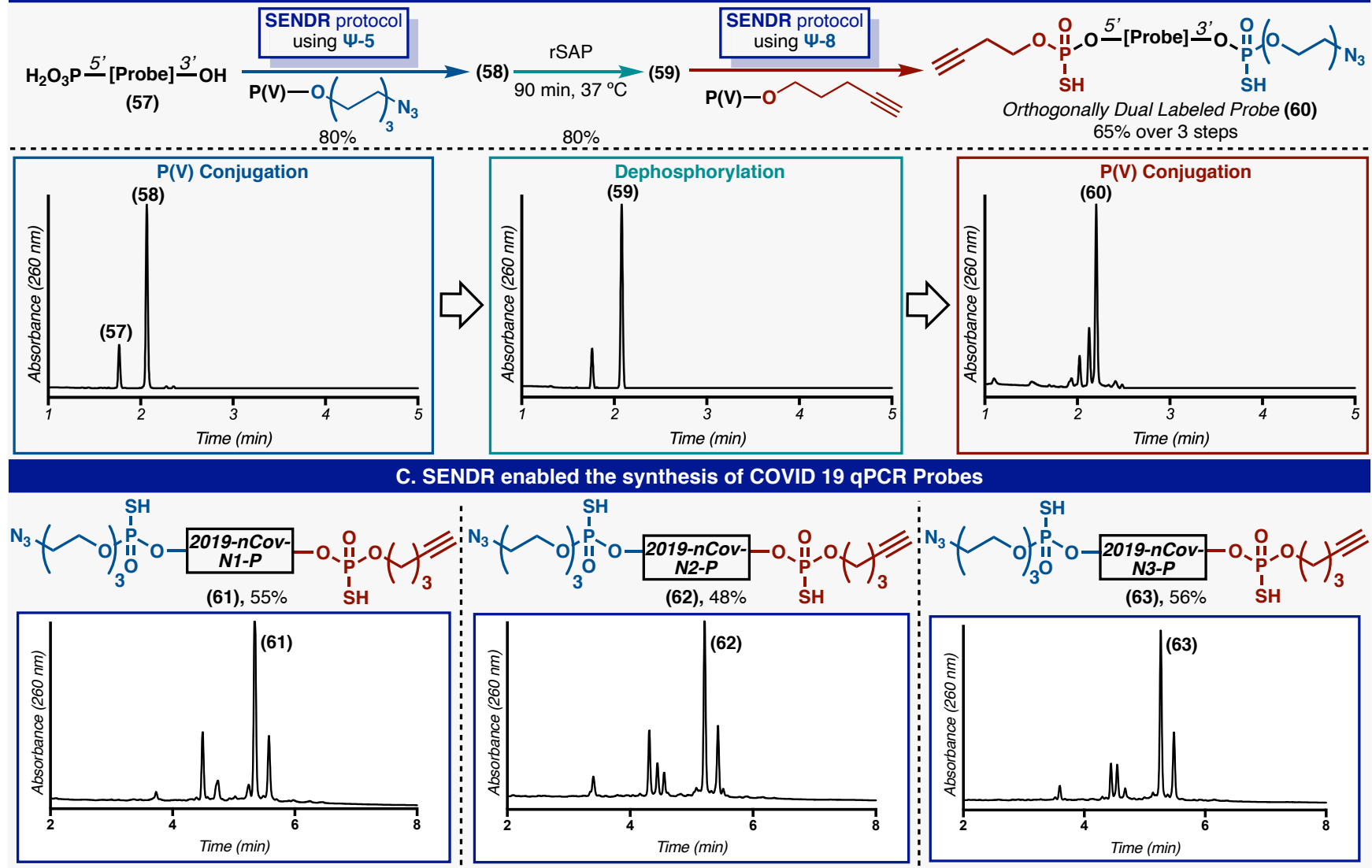

Figure 7: Creation of dual labeled DNA probes. (A) Construction of dual labeled probes from cheap precursors. (B) The synthesis of a positive control TaqMan probe for RNaseP. (C) Synthesis of the COVID 19 qPCR panel of probes. 


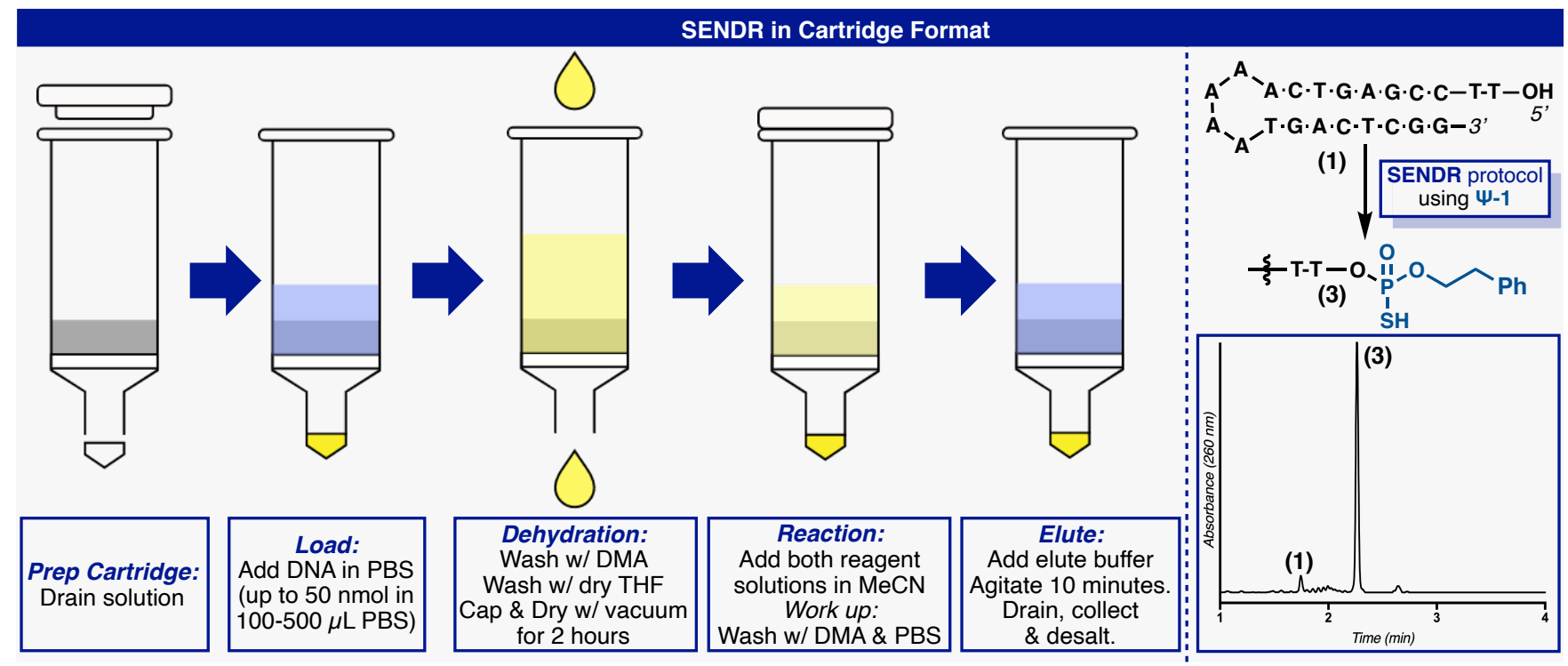

Figure 8: Graphical workflow of SENDR in kit format.

From a pragmatic standpoint, a simple kit-format would be of use to the community. Towards that end, a "SENDR kit" was created from readily-available consumables (Figure 8). The SENDR process is simple and robust enough to be miniaturized and performed in a cartridge/flow set up and all reagents employed are shelf stable indefinitely. Gratifyingly, when performed in a cartridge, the process proved simpler and faster than the previously-employed microcentrifuge tubes. Also, the handling procedures were greatly simplified and could be performed by any researcher with basic micropipetting skills. Importantly, reaction efficiency was identical to reactions performed in microcentrifuge tubes (see SI). Using this kit, a researcher could customize synthetic or biochemically-derived DNA, in-house, with a suite of commercialized reagents. We believe that SENDR kits will expand the toolbox and allow researchers to pursue experimental designs that were previously out of reach. We envision that SENDR will democratize site specific DNA modifications. More broadly, the modular nature of the process could permit a more medicinal chemistry mindset into the derivatization of complex DNA-based conjugates. This initial disclosure demonstrates conjugations that are compatible with simple organic molecules, proteins, aptamers, and ASOs. Numerous extensions such as applications to carbohydrate conjugation, and multiplexed high throughput arrays, could be anticipated.

\section{AUTHOR INFORMATION}

\section{Corresponding Authors}

*pbaran@scripps.edu

*dawson@scripps.edu

\section{ACKNOWLEDGMENT}

Financial support for this work was NIH (GM-132787 for P.E.D and GM-118176 P.S.B). The authors thank Christoph Rader and Junpeng Qi for graciously proving DVD IgG. DTF was supported by the National Center for Advancing Translational Sciences, National Institutes of Health, through grant number UL1 TR002551 and linked award TL1 TR002551. The content is solely the responsibility of the authors and does not necessarily represent the official views of the NIH. All nonphotorealistic biomolecular illustrations were created with Goodsell's Illustrate. ${ }^{68}$ Authors are grateful to Dr. Dee-Hua Huang and Dr.
Laura Pasternack (Scripps Research) for assistance with nuclear magnetic resonance (NMR) spectroscopy.

\section{REFERENCES}

1. D. M. Kolpashchikov, Acc. Chem. Res. 2019, 52, 1949-1956.

2. D. J. Nieves, K. Gaus and M. A. B. Baker, Genes (Basel) 2018, 9.

3. R. Jungmann, M. S. Avendano, J. B. Woehrstein, M. Dai, W. M. Shih and P. Yin, Nat. Methods 2014, 11, 313-318.

4. R. Jungmann, C. Steinhauer, M. Scheible, A. Kuzyk, P. Tinnefeld and F. C. Simmel, Nano Lett. 2010, 10, 4756-4761.

5. J. Schnitzbauer, M. T. Strauss, T. Schlichthaerle, F. Schueder and R. Jungmann, Nat. Protoc. 2017, 12, 1198-1228.

6. P. M. Holland, R. D. Abramson, R. Watson and D. H. Gelfand, Proc. Natl. Acad. Sci. U.S.A. 1991, 88, 7276-7280.

7. E. Navarro, G. Serrano-Heras, M. J. Castano and J. Solera, Clin. Chim. Acta. 2015, 439, 231-250.

8. M. J. Espy, J. R. Uhl, L. M. Sloan, S. P. Buckwalter, M. F. Jones, E. A. Vetter, J. D. C. Yao, N. L. Wengenack, J. E. Rosenblatt, F. R. Cockerill and T. F. Smith, Clin. Microbiol. Rev. 2006, 19, 165-256.

9. G. Mayer, J. Muller and C. E. Lunse, Wiley Interdiscip. Rev. RNA 2011, 2, 32-41.

10. R. A. Cardullo, S. Agrawal, C. Flores, P. C. Zamecnik and D. E. Wolf, Proc. Natl. Acad. Sci. U.S.A. 1988, 85, 8790-8794.

11. L. E. Morrison, T. C. Halder and L. M. Stols, Analytical Biochemistry 1989, 183, 231-244.

12. B. D. Hall and S. Spiegelman, Proc. Natl. Acad. Sci. U.S.A. 1961, 47, 137-163.

13. E. T. Bolton and C. B. Mc, Proc. Natl. Acad. Sci. U.S.A. 1962, 48, 1390-1397.

14. D. M. Kolpashchikov, Scientifica (Cairo), 2012, 2012, 928783.

15. S. Tyagi and F. R. Kramer, Nat. Biotechnol. 1996, 14, 303308.

16. E. M. Southern, J. Mol. Biol. 1975, 98, 503-517.

17. C. G. Kevil, L. Walsh, F. S. Laroux, T. Kalogeris, M. B. Grisham and J. S. Alexander, Biochem. Biophys. Res. Commun. 1997, 238, 277-279.

18. G. H. Keller, C. U. Cumming, D.-P. Huang, M. M. Manak and R. Ting, Anal. Biochem. 1988, 170, 441-450.

19. H. Sakamoto, F. Traincard, T. Vo-Quang, T. Ternynck, J.-L. Guesdon and S. Avrameas, Mol. Cell. Probe. 1987, 1, 109-120. 
20. T. Tasara, B. Angerer, M. Damond, H. Winter, S. Dorhofer, U. Hubscher and M. Amacker, Nucleic Acids Res. 2003, 31, 2636-2646.

21. N. Paul and J. Yee, BioTechniques 2010, 48, 333-334.

22. R. K. Ralph, R. J. Young and H. G. Khorana, J. Am. Chem. Soc. 1962, 84, 1490-1491.

23. B. C. Chu, G. M. Wahl and L. E. Orgel, Nucleic Acids Res. 1983, 11, 6513-6529.

24. B. C. Chu and L. E. Orgel, Nucleic Acids Res. 1988, 16, 36713691.

25. S. S. Ghosh, P. M. Kao and D. Y. Kwoh, Anal. Biochem. 1989, 178, 43-51.

26. S. S. Ghosh, P. M. Kao, A. W. McCue and H. L. Chappelle, Bioconjug. Chem. 1990, 1, 71-76.

27. D. Xu, N. Rivas-Bascon, N. M. Padial, K. W. Knouse, B. Zheng, J. C. Vantourout, M. A. Schmidt, M. D. Eastgate and P. S. Baran, J. Am. Chem. Soc. 2020, 142, 5785-5792.

28. K. W. Knouse, J. N. deGruyter, M. A. Schmidt, B. Zheng, J. C. Vantourout, C. Kingston, S. E. Mercer, I. M. McDonald, R. E. Olson, Y. Zhu, C. Hang, J. Zhu, C. Yuan, Q. Wang, P. Park, M. D. Eastgate and P. S. Baran, Science, 2018, 361, 1234-1238.

29. D. T. Flood, C. Kingston, J. C. Vantourout, P. E. Dawson and P. S. Baran, Isr. J. Chem. 2020, 60, 1-14

30. A. Arcella, G. Portella, R. Collepardo-Guevara, D. Chakraborty, D. J. Wales and M. Orozco, J. Phys. Chem. B. 2014, 118, 8540-8548.

31. D. T. Flood, S. Asai, X. Zhang, J. Wang, L. Yoon, Z. C. Adams, B. C. Dillingham, B. B. Sanchez, J. C. Vantourout, M. E. Flanagan, D. W. Piotrowski, P. Richardson, S. A. Green, R. A. Shenvi, J. S. Chen, P. S. Baran and P. E. Dawson, J. Am. Chem. Soc. 2019, 141, 9998-10006.

32. D. T. Flood, N. L. Yan and P. E. Dawson, Angew. Chem. Int. Ed. 2018, 57, 8697-8701.

33. D. T. Flood, X. Zhang, X. Fu, Z. Zhao, S. Asai, B. B. Sanchez, E. J. Sturgell, J. C. Vantourout, P. Richardson, M. E. Flanagan, D. W. Piotrowski, D. K. Kolmel, J. Wan, M. H. Tsai, J. S. Chen, P. S. Baran and P. E. Dawson, Angew. Chem. Int. Ed. 2020, DOI: 10.1002 anie.201915493.

34. P. A. Cistrone and P. E. Dawson, ACS Comb. Sci. 2016, 18, 139-143.

35. Y. Li, S. Chen, N. Liu, L. Ma, T. Wang, R. N. Veedu, T. Li, F. Zhang, H. Zhou, X. Cheng and X. Jing, Biotechniques. 2020, DOI: $10.2144 /$ btn-2019-0109.

36. E. M. Sletten and C. R. Bertozzi, Angew. Chem. Int. Ed. 2009 , 48, 6974-6998.

37. N. K. Devaraj, ACS Cent. Sci. 2018, 4, 952-959.

38. N. J. Agard, J. A. Prescher and C. R. Bertozzi, J. Am. Chem. Soc. 2004, 126, 15046-15047.

39. V. V. Rostovtsev, L. G. Green, V. V. Fokin and K. B. Sharpless, Angew. Chem. Int. Ed. 2002, 41, 2596-2599.

40. H. Staudinger and J. Meyer, Helv. Chim. Acta. 1919, 2, 635646.

41. M. Honcharenko, D. Honcharenko and R. Stromberg, Curr. Protoc. Nucleic Acid Chem. 2020, 80, e102.

42. C. M. Perry and J. A. Balfour, Drugs 1999, 57, 375-380; discussion 381.

43. C. A. Stein and D. Castanotto, Mol. Ther. 2017, 25, 10691075.

44. R. Stoltenburg, P. Krafcikova, V. Viglasky and B. Strehlitz, Sci. Rep. 2016, 6, 33812.

45. C. Tuerk and L. Gold, Science 1990, 249, 505-510.

46. C. Chai, Z. Xie and E. Grotewold, Methods Mol. Biol. 2011, 754, 249-258.

47. M. Synakewicz, D. Bauer, M. Rief and L. S. Itzhaki, Sci. Rep. 2019, 9, 13820.

48. G. Bernardinelli and B. Hogberg, Nucleic Acids Res. 2017, 45, e160.
49. X. Yan, H. Zhang, Z. Wang, H. Peng, J. Tao, X. F. Li and X. Chris Le, Chem. Commun. 2018, 54, 7491-7494.

50. C. M. Niemeyer, Angew. Chem. Int. Ed. 2010, 49, 1200-1216.

51. A. W. Feldman, V. T. Dien, R. J. Karadeema, E. C. Fischer, Y. You, B. A. Anderson, R. Krishnamurthy, J. S. Chen, L. Li and F. E. Romesberg, J. Am. Chem. Soc. 2019, 141, 10644-10653.

52. Y. Won, H. Jeon, A. D. Pagar, M. D. Patil, S. P. Nadarajan, D. T. Flood, P. E. Dawson and H. Yun, Chem. Commun. 2019, 55, 15133-15136.

53. D. Xie, C. Yao, L. Wang, W. Min, J. Xu, J. Xiao, M. Huang, B. Chen, B. Liu, X. Li and H. Jiang, Antimicrob. Agents. Chemother. 2010, 54, 191-196.

54. J. Feng, C. Zhao, L. Wang, L. Qu, H. Zhu, Z. Yang, G. An, H. Tian and C. Shou, Theranostics 2018, 8, 2094-2106.

55. K. Sasaki, J. Ishihara, A. Ishihara, R. Miura, A. Mansurov, K. Fukunaga and J. A. Hubbell, Sci. Adv. 2019, 5, eaaw6081.

56. H. J. Byeon, S. Y. Min, I. Kim, E. S. Lee, K. T. Oh, B. S. Shin, K. C. Lee and Y. S. Youn, Bioconjug. Chem. 2014, 25, 2212-2221.

57. K. Thibaudeau, R. Leger, X. Huang, M. Robitaille, O. Quraishi, C. Soucy, N. Bousquet-Gagnon, P. van Wyk, V. Paradis, J. P. Castaigne and D. Bridon, Bioconjug. Chem. 2005, 16, 1000-1008.

58. M. A. Gertz, M. Scheinberg, M. Waddington-Cruz, S. B. Heitner, C. Karam, B. Drachman, S. Khella, C. Whelan and L. Obici, Expert Rev. Clin. Pharmacol. 2019, 12, 701-711.

59. M. D. Benson, M. Waddington-Cruz, J. L. Berk, M. Polydefkis, P. J. Dyck, A. K. Wang, V. Plante-Bordeneuve, F. A. Barroso, G. Merlini, L. Obici, M. Scheinberg, T. H. Brannagan, 3rd, W. J. Litchy, C. Whelan, B. M. Drachman, D. Adams, S. B. Heitner, I. Conceicao, H. H. Schmidt, G. Vita, J. M. Campistol, J. Gamez, P. D. Gorevic, E. Gane, A. M. Shah, S. D. Solomon, B. P. Monia, S. G. Hughes, T. J. Kwoh, B. W. McEvoy, S. W. Jung, B. F. Baker, E. J. Ackermann, M. A. Gertz and T. Coelho, N. Engl. J. Med. 2018, 379, 22-31.

60. L. Gales, Pharmaceuticals (Basel) 2019, 12.

61. A. R. Nanna and C. Rader, Methods Mol. Biol. 2019, 2033, $39-52$.

62. A. R. Nanna, X. Li, E. Walseng, L. Pedzisa, R. S. Goydel, D. Hymel, T. R. Burke, Jr., W. R. Roush and C. Rader, Nat. Commun. 2017, 8, 1112.

63. C. Rader, J. M. Turner, A. Heine, D. Shabat, S. C. Sinha, I. A. Wilson, R. A. Lerner and C. F. Barbas, J. Mol. Bio. 2003, 332, 889-899.

64. J. Wagner, R. A. Lerner and C. F. Barbas, 3rd, Science, 1995, 270, 1797-1800.

65. J. L. Millan, Purinergic Signal. 2006, 2, 335-341.

66. V. M. Corman, O. Landt, M. Kaiser, R. Molenkamp, A. Meijer, D. K. W. Chu, T. Bleicker, S. Brunink, J. Schneider, M. L. Schmidt, D. Mulders, B. L. Haagmans, B. van der Veer, S. van den Brink, L. Wijsman, G. Goderski, J. L. Romette, J. Ellis, M. Zambon, M. Peiris, H. Goossens, C. Reusken, M. P. G. Koopmans and C. Drosten, Euro. Surveill. 2020, 25.

67. Research Use Only 2019-Novel Coronavirus (2019-nCoV) Real-time RT-PCR Primer and Probe Information, https://www.cdc.gov/coronavirus/2019-ncov/lab/rt-pcr-panelprimer-probes.html).

68. D. S. Goodsell, L. Autin and A. J. Olson, Structure 2019, 27, 1716-1720. 